\title{
PERFORMANCE ANALYSIS AND SOFT DEMAPPING FOR CODED MIMO-OFDM SYSTEMS
}

\author{
Mahmoud Abdullahi, Pei Xiao \\ Institute of Communication Systems (ICS), Home of 5G Innovation Centre \\ University of Surrey, Guildford, Surrey, UK \\ Email: \{m.abdullahi, p.xiao\}@ surrey.ac.uk
}

\begin{abstract}
Multiple input multiple output-Orthogonal frequency division multiplexing (MIMO-OFDM) is a viable solution for providing high data rate services in harsh channel environments. The optimum receivers for them are those based on the maximum likelihood criterion. However, they have a prohibitive complexity especially when channel dimensions are high and coding is employed. Zero Forcing (ZF) and Linear Minimum Mean Square Error (MMSE) receivers on the other hand provide practicable and low complexity solutions for detection, but require soft demappers to deduce the soft bits information contained in each of the received symbols. In this work, we present the $\mathrm{ZF}$ and MMSE receiver analysis of a bit interleaved and coded MIMO-OFDM system and propose a soft output demapper based on MMSE equalizer output to demap the information needed for viterbi decoding. A comparison of the proposed soft demapper with conventional soft demappers in literature show a significant performance improvement. We also noticed that it is more advantageous to apply the proposed demapper on a MIMO-OFDM system employing higher modulation schemes.
\end{abstract}

\section{INTRODUCTION}

Multiple Input Multiple Output (MIMO) technology combined with multicarrier modulation provides high data rate transmissions resulting from spatial multiplexing gain (between spatially seperated antennas at both the transmitter and receiver) and mitigation of the effect of frequency selective distortion in a richly scattered environment. The state-ofthe-art multicarrier modulation techniques include orthogonal frequency division multiplexing (OFDM) [1], filtered OFDM [2], universal filtered multi-carrier (UFMC) [3] [4], filter bank multi-carrier (FBMC) [5] [6] etc. In this paper, we study the synergistic combination of MIMO and OFDM techniques. Wireless LAN systems such as IEEE 802.11n [7], IEEE 802.11ac [8] and cellular systems such as 3GPP-LTE are exemplary cases to which MIMO-OFDM has been applied. To improve error performance due to fast fading, forward error correction (FEC) coding and bit interleaving are incorporated which is referred to as bit interleaved coded and modulated (BICM) MIMO-OFDM systems. The optimum receiver for this system is the one based on maximum likelihood detection but is very complex to implement in practice as its complexity increases exponentially with the number of transmitter antennas and the constellation size [9]. A more practical alternative is to employ linear equalizers to compensate channel fading and then pass the output to a soft demapper to extract the soft bits before de-interleaving and subsequent decoding.

Conventional soft demappers have been derived based on the assumption that equalizer outputs can be modelled as random variables with Gaussian distributions. [10] [11] derived the soft bits for MMSE-MIMO system as a function of signal to interference plus noise ratio (SINR) of the equalized symbols.

In this paper, we derived a new soft demapper that estimates the variance of coloured noise as a function of the conditional mean of the equalizer outputs. This method would add a little more complexity to the conventional soft demapper by computing the mean and variance of the equalized symbols but achieves significant performance improvement.

The remainder of this paper is organised as follows: Sec. II presents the MIMO-OFDM system model. Sec. III analyses the $\mathrm{ZF}$ receiver and Sec. IV analyses MMSE receiver with the derivation of mean and variance of the equalizer output. In Sec. V, we derived the proposed MMSE soft demapper and also give the expression for conventional demapper. Sec. VI presents simulation results to compare the proposed soft demapper with the conventional method and we finally conclude the paper in Sec. VII.

\section{System MODEL}

The system architecture for a BICM MIMO-OFDM transmitter is illustrated in Fig. 1. The input data bits are convolutionally encoded, interleaved and modulated using Gray-label mapping to produce complex $2^{m}-$ Mary QAM modulation symbols, each corresponding to $b_{1}, b_{2}, \ldots . b_{m}$ bits sequence. A demultiplexer is then used to create $N_{t}$ spatial streams of the modulated symbols. Each of the stream is passed through an IFFT block for mapping across OFDM subcarriers. To form an OFDM block, a cyclic prefix longer than the channel delay spread is inserted to mitigate the effect of both ISI and ICI. The complex OFDM symbols given by $X(k)=X_{r}(k)+X_{q}(k)$ are assumed to be independent and identically distributed with zero mean and unit variance. The total transmit power is distributed equally over the transmit antennas. $X_{i}(k)$ represents the $k^{t h}$ subcarrier symbol to be transmitted on the $i^{t h}$ transmit antenna of the $N_{t} \mathrm{x} 1$ antenna 
dimensions. In the receiver side shown in Fig.2, $N_{r} \mathrm{x} 1$ antennas are used to receive signals each comprising copies of symbols from the other transmit antennas. To recover the original signal transmitted from the transmitter after timing and frequency synchronisation, the cyclic prefix is removed and then FFT is performed to convert the time domain signals to frequency domain symbols. The output of the FFT-block at the $k^{t h}$ subcarrier is given by

$$
\mathbf{y}(k)=\mathbf{H}(k) \mathbf{x}(k)+\mathbf{n}(k),
$$

where $\mathbf{y}(k)=\left[y_{1}(k), y_{2}(k), \ldots .\right]^{T} \in C^{N_{r} x 1}$ is a column vector of received data symbols. $\mathbf{x}(k)=\left[x_{1}(k), x_{2}(k) \ldots \ldots . .\right]^{T}$ is a also a column vector of QAM symbols transmitted across $N_{t}$ antennas at the $k^{t h}$ subcarrier with $E\left[\left|x_{i}\right|^{2}\right]=\sigma_{x}^{2} . \mathbf{n}(k)=$ $\left[n_{1}(k), n_{2}(k), \ldots . .\right]^{T} \in C^{N_{r} x 1}$ is a complex additive white Gaussian noise vector which is due to thermal noise at the receiver, the element of which are independent and identically distributed with zero mean and covariance $E\left[\left|n n^{*}\right|\right]=\sigma_{n}^{2}$. $\mathbf{H}(k)$ is the $N_{r} \mathrm{x} N_{t}$ MIMO channel frequency response matrix of the $k^{t h}$ subcarrier. The elements of $\mathbf{H}(k)$ are also assumed to be independent and identically distributed (i.i.d) zero mean circularly symmetric complex Gaussian with $\sum h_{j, i}=1$ and given by

$$
\mathbf{H}(k)=\left[\begin{array}{cccc}
h_{j, 1} & h_{j, 2} & \ldots & h_{j, N_{t}} \\
\vdots & \vdots & \vdots \\
h_{N_{r}, 1} & h_{N_{r}, 2} & \ldots & h_{N_{r} N_{t}}
\end{array}\right]
$$

Expanding Eq.(1) yields

$$
y_{j}=\sum_{i=1}^{N_{t}}\left(h_{j i} x_{i}\right)+n_{j} .
$$

The above expression shows that each receiver antenna gets a copy of symbols transmitted from all transmit antennas. Consequently, the system suffers from multi-antenna interference in addition to channel fading, necessitating equalization to remove those effects.

\section{ZERO FORCING RECEIVER}

The zero forcing equalizer aims to cancel the channel frequency response on the symbol of interest and forces all interferences of other symbols from neighbouring antennas to zero. $\mathrm{ZF}$ weight matrix is given as:

$$
\mathbf{W}=\left(\mathbf{H}^{H} \mathbf{H}\right)^{-1} \mathbf{H}^{H}
$$

The output of the equalizer is then given by

$$
\mathbf{w}^{H}\left(\mathbf{h}_{i} x_{i}+\mathbf{n}\right)
$$

where $\mathbf{z}$ is the equalized vector. The equalizer output for the $i^{t h}$ stream ( $i^{t h}$ transmitter) in the $k^{t h}$ subcarrier can be expressed as:

$$
z_{i}(K)=\mathbf{w}_{i}(K) \mathbf{h}_{i}(K) x_{i}(K)+\mathbf{w}_{i}(K) n(K)
$$

where $\mathbf{w}_{i}(k)$ and $\mathbf{h}_{i}(k)$ are the $i^{t h}$ row vector of $\mathbf{W}$ and $i^{t h}$ column vector of $\mathbf{H}$ matrices in the K-subcarrier respectively. Eqn.6 can further be written as

$$
z_{i}(K)=x_{i}(K)+v(K),
$$

which is approximated by a complex Gaussian distributed random variable. The mean $\mu_{z i}$ can be estimated as $E\left[z_{i}\right]=x_{i}$ and the variance of $z_{i}$, can be computed as

$$
\nu_{z i}^{2}=E\left[\nu \nu^{*}\right]=\|\mathbf{w}\|^{2} \sigma_{n}^{2}
$$

\section{Minimum Mean Square Error (MMSE) RECEIVER}

The MIMO receiver separates the received vector into $N_{r}$ parallel streams which can be modelled as [12]

$$
y=h_{i} x_{i}+\sum_{k \neq i} h_{k} x_{k}+n,
$$

where $y$ is a combination of symbol of interest $x_{i}$ which has gone through channel fading, ISI component from other transmitting antennas and the additive white Gaussian noise. A linear MMSE receiver on the other hand decouples the MIMO channel into $N_{t}$ parallel streams representing estimated symbols from the transmitting antennas. The MMSE weight matrix is given as

$$
\mathbf{W}=\left(\mathbf{H}^{H} \mathbf{H}+N_{0} \mathbf{I}\right)^{-1} \mathbf{H}^{H}
$$

where $\mathbf{I}$ is an identity matrix of dimension $N_{r}$. The output of the equalizer is then given by

$$
\mathbf{z}=\mathbf{w}^{H}\left(\mathbf{h}_{i} x_{i}+\sum_{k \neq n} \mathbf{h}_{k} x_{k}+\mathbf{n}\right)
$$

where $\mathbf{z}$ is the equalized vector. The equalizer output for the $i^{t h}$ stream ( $i^{\text {th }}$ transmitter) in the $k^{\text {th }}$ subcarrier can be expressed as

$z_{i}(K)=\mathbf{w}_{i}(K)\left(\mathbf{h}_{i}(K) x_{i}(K)+\sum_{k \neq n} \mathbf{h}_{k}(K) x_{k}(K)+\mathbf{n}(K)\right)$

where $\mathbf{w}_{i}(k)$ and $\mathbf{h}_{i}(k)$ are the $i^{t h}$ row vector of $\mathbf{W}$ and $i^{t h}$ column vector of $\mathbf{H}$ matrices in the K-subcarrier respectively.

The output of the MMSE equalizer $\left(z_{i}\right)$ is also approximated by a complex Gaussian distributed random variable with mean $\mu_{z i}$ and coloured noise variance $\nu_{z i}^{2}$.

$$
p\left(z_{i} \mid x_{i}, h_{i}\right) \sim C N\left(\mu_{z i}, \nu_{z i}^{2}\right) .
$$

To compute the mean $\mu_{z i}$ using Eqn.(12), we have:

$$
\begin{aligned}
& \mu_{z i}=E\left[z_{i} \mid x_{i}, H\right]=w_{i}^{H}\left(E\left[y \mid x_{i}, H\right]\right) \\
& =w_{i}\left(x_{i} h_{i}-E\left[\sum_{k \neq i} x_{k} h_{k} \mid x_{i}, H\right]\right)
\end{aligned}
$$

where $\alpha$ is given by

$$
\alpha=E\left[\sum_{k \neq i} x_{k} / x_{i}\right] .
$$


By substituting $\alpha$ as shown in appendix,

$$
\left.\mu_{z i}=w_{i}\left(x_{i} h_{i}-\sqrt{(} N_{t}-1\right) x_{i} h_{k}\right)
$$

where $N_{t}$ is the number of transmitting antennas.

For the variance $\nu_{z i}^{2}=\operatorname{Var}\left[z_{i} \mid x_{i}, H\right]$,

$$
\nu_{z i}^{2}=E\left[\left(z_{i}-\mu_{z i}\right)\left(z_{i}-\mu_{z i}\right)^{*} \mid x_{i}, H\right]
$$

where $\left.q=w_{i}\left(h_{i}-\sqrt{(} N_{t}-1\right) h_{k}\right)$ from Eqn.(16). we know from Eqn.(11) that $z=w^{H} \cdot y$ and also $w^{H}=R_{y y}^{-1} P_{x y}$ in [13]. Since, $E\left[z_{i} \mid x_{i}, H\right]=q x_{i}$, then

$$
E\left[z_{i} x_{i} \mid x_{i}, H\right]=q E\left[x_{i} x_{i}\right]
$$

Therefore,

$$
\begin{aligned}
& \nu_{z i}^{2}=E\left[w R_{y y} w^{H}-2 q^{2} x_{i} x_{i}+q^{2} x_{i} x_{i}\right] \\
& =q E_{x}-q^{2} E_{x}
\end{aligned}
$$

\section{SOFT OUTPUT DEMAPPING}

Since we can estimate the mean and variance of the equalized symbols given in Eqns.(8)(16)\&(19), the LLR of the $n^{\text {th }}$ bit of the equalized symbols can then be computed from the conditional pdf of $z_{i}$ which is given as

$$
p(z \mid x=\beta)=\frac{1}{\sqrt{2 \pi \sigma}} \exp \left\{-\frac{1}{2} \frac{|z-\beta|^{2}}{\sigma^{2}}\right\},
$$

where $\beta$ is the amplitude of the transmitted symbol on the QAM constellation. The LLR function is given by [14] as:

$$
\wedge\left(b_{n}\right)=\log \left[\frac{p\left(b_{n}=1 \mid z\right)}{\left(p\left(b_{n}=0 \mid z\right)\right.}\right] .
$$

By applying bayes theorem and assuming bits are equiprobable, $b_{n}$ can be re-written as

$$
\wedge\left(b_{n}\right)=\log \left[\frac{\sum p\left(z \mid b_{n}=\beta_{1}\right)}{\sum p\left(z \mid b_{n}=\beta_{0}\right)}\right]
$$

The above equation can be approximated using the max-log-map function which can also be expressed as min-euclidean distance function as [14]

$$
\wedge\left(b_{n}\right)=\frac{1}{\nu^{2}}\left\{\min \left|z_{i}-\beta_{1}\right|^{2}-\min \left|z_{i}-\beta_{0}\right|^{2}\right\}
$$

\section{CASE I: Conventional Soft Demapper}

The conventional soft demapper is implemented with the assumption that noise variance is the same in all the equalized symbols and expressed by [15] as,

$$
\wedge\left(b_{n}\right)=\frac{1}{\sigma_{n}^{2}}\left\{\min \left|z_{i}-\beta_{1}\right|^{2}-\min \left|z_{i}-\beta_{0}\right|^{2}\right\} .
$$

Because of noise colouration as a result of equalization, the performance of conventional demapper above can be enhanced in OFDM systems [16] by appropriately scaling the soft demapper using the variance of coloured noise. This was derived with the assumption that zero forcing equalization is optimum for OFDM systems as individaul subcarriers experience narrow-band fading.

$$
\wedge\left(b_{n}\right)=\|\mathbf{w}\|^{2}\left\{\min \left|z_{i}-\beta_{1}\right|^{2}-\min \left|z_{i}-\beta_{0}\right|^{2}\right\}
$$

\section{CASE III: Proposed Soft Demapper}

This demapper is proposed for MMSE receivers to take into consideration the estimated mean and variance of the received symbols. According to the derivations in Eqns. (16)\&(19), the proposed soft demapper can be expressed as:

$$
\begin{aligned}
& \wedge\left(b_{n}\right)=\frac{q_{z}}{\nu_{z}^{2}}\left\{\min \left|z_{i}-q \beta_{1}\right|^{2}-\min \left|z_{i}-q \beta_{0}\right|^{2}\right\} \\
& \quad=\frac{1}{1-w_{i}\left(h_{i}-\sqrt{(} t-1\right) h_{k}}\left\{\min \mid z_{i}-w_{i}\left(h_{i}-\right.\right. \\
& \left.\quad \sqrt{(} t-1) h_{k}\right)\left.\beta_{1}\right|^{2}-\min \mid z_{i}-w_{i}\left(h_{i}-\sqrt{(} t-1\right) \\
& \left.\left.h_{k}\right)\left.\beta_{0}\right|^{2}\right\}
\end{aligned}
$$

Note that this soft demapping approach has an increasing computational complexity with constellation size and transmit antennas therefore we further simplify the algorithm to maintain approximately the same complexity according to [17].

Consider a QAM constellation with $d_{n}$ as half the distance between the partition boundaries relative to $b_{n}$ for $n>1$ (see appendix). Then the general simplified LLR function can be approximated as:

$$
\begin{gathered}
D_{n} \simeq \begin{cases}z_{i} & n=1 \\
-\left|D_{n-1}\right|+q d_{n} & n>1\end{cases} \\
\wedge\left(b_{n}\right)=\frac{q}{\nu^{2}} D_{n} \quad n \geq 1
\end{gathered}
$$

By substituting the $q$ and $\nu^{2}$ into Eqn. (28), the simplified $n^{\text {th }}$ bit function can be expressed as:

$$
\wedge\left(b_{n}\right)=\frac{1}{1-w_{i}\left(h_{i}-\sqrt{(} t-1\right) h_{k}} D_{n} \quad n \geq 1 .
$$

Equation (29) represents simplified LLR function that takes into consideration the effects of ISI and coloured noise due to filtering.

\section{Simulations And Result}

In order to evaluate the performance of the proposed MMSE-based soft demapper for BICM-MIMO-OFDM systems, we simulate the conventional demapper in CASE I using both ZF and MMSE estimates and compare the performance with CASE II and CASE III. In the simulations, we used a $1 / 2$ rate convolutional encoder with a constraint length of 7 ,

\section{CASE II: Scaled Soft Demapper}




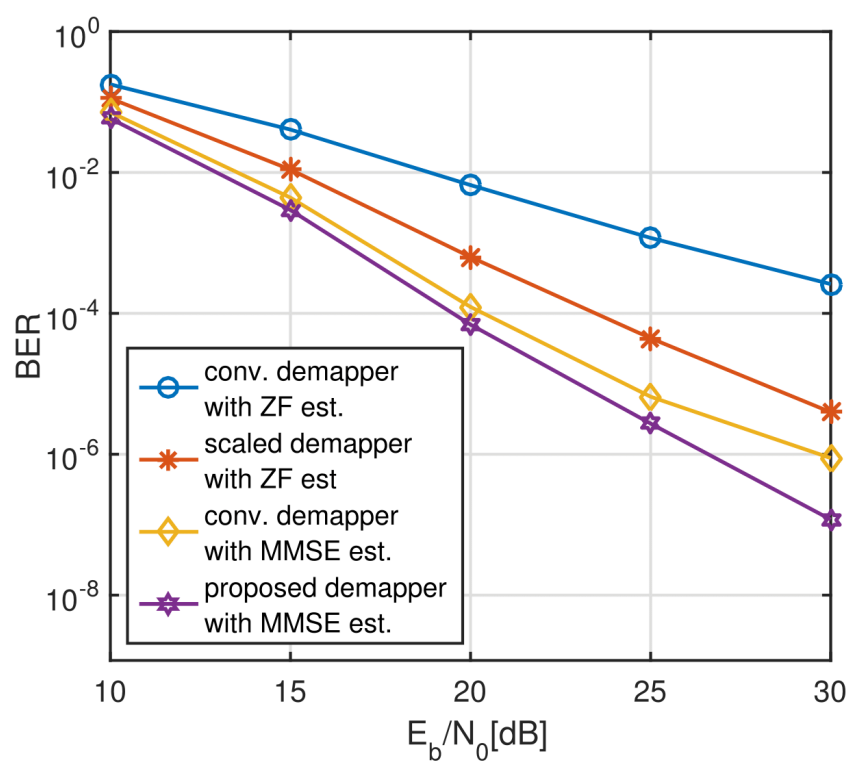

Fig. 1. BER performance of 2x2-16QAM-BICM-MIMO-OFDM system

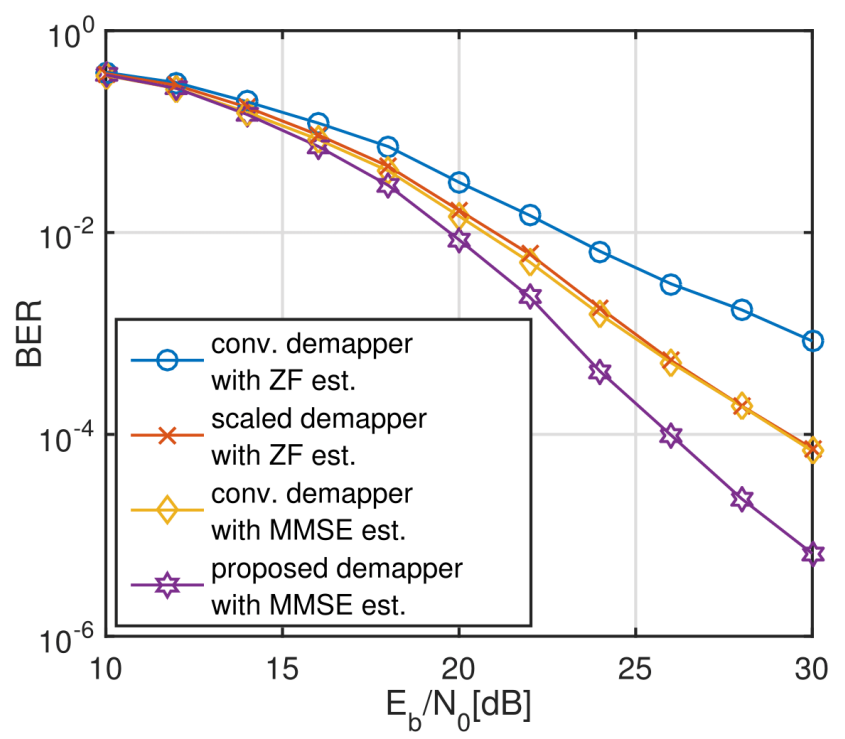

Fig. 2. BER performance of 2X2-256QAM-BICM-MIMO-OFDM system

generator polynomial of $(131,171)_{8}$, and a random interleaver. The FFT size that produced the OFDM symbols was choosen to be 256 and the length of $\mathrm{CP}$ is 16 . We used the channel model adopted by $I E E E 802.11$ working group in [18] to generate the channel fading between each $j^{t h}$ receiver and $i^{\text {th }}$ transmitter.

The output of a linear MMSE equalizer is an estimate of the transmitted symbol which is affected by coloured Gaussian noise and needs to be demapped into the component bits that made up the transmitted symbol. We derive a soft bit demapper with the approriate scaling coefficients $q$ and $\nu^{2}$ in Eqn.27. To reduce the complexity involved in computing

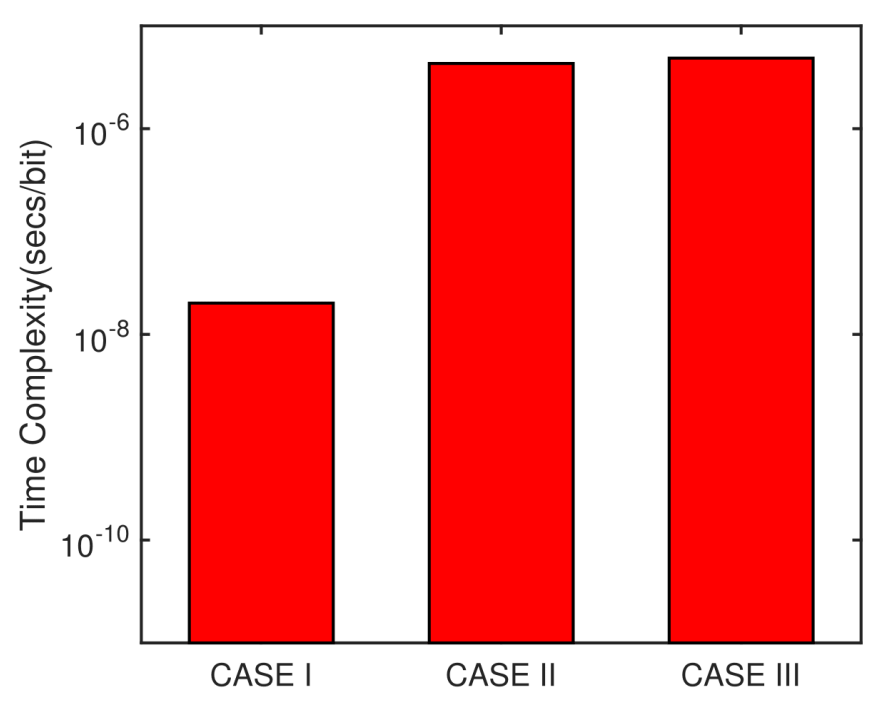

Fig. 3. Complexity Comparison

minimum euclidean distance which grows exponentially with the modulation order, we simplify the algorithm to maintain its computational complexity even with higher modulation in Eqns. (27)\&(29) and apply it to 16QAM and 256QAM modulation levels, and 2X2 MIMO dimension.

BER versus $E_{b} / N_{0}$ plots in Fig. 3 and Fig. 4 both show superiority of Case II (ZF- Noise Scaled) and Case III (Proposed MMSE soft demapper) over Case I (Conventional soft demapper) in all the modulation levels used. We noticed a more distinct power reduction by our proposed scheme compared to conventional demapper in 256QAM MIMO-OFDM than in 16QAM MIMO-OFDM e.g., for 2X2 MIMO-OFDM system at BER of $10^{-4}$, our proposed scheme shows an improvement of $3.5 \mathrm{~dB}$ over conventional method in 256QAM against $0.8 \mathrm{~dB}$ improvemnet in 16QAM. Fig. 5 compares the computational time of all the algorithms which indicates the computational complexity to give a base line for trade-off analysis.

\section{CONCLUSION}

We have analysed linear ZF and MMSE receivers for BICM-MIMO-OFDM systems and derived a soft-demapper based on the conditional mean and variance of the equalizer output. In order to reduce the increasing complexity associated with increasing order of modulation for minimum euclidean distance metric, we approximated the soft demapper with an expression that fairly maintains the same computational complexity for all practicable QAM modulation. A BER performance comparison of the proposed soft demapper, a ZF scaled demapper and a conventional soft demapper from the literature confirms superiority of the proposed scheme especially at higher modulation order. This justifies the viability of the proposed soft demapper for practical low dimensioned MIMO systems with high order modulation schemes.

\section{APPENDIX A}


PROOF OF $\alpha$ IN Equation(16)

$$
\alpha=E\left[\sum_{k \neq i} x_{k} / x_{i}\right] .
$$

by taking the square of both sides,

$$
\begin{aligned}
\alpha .^{2} & =E\left[\sum_{k \neq i} x_{k} / x_{i}\right]^{2} . \\
& =\left(N_{t}-1\right) E_{x} / E_{x}
\end{aligned}
$$

Since $E_{x}=1$ (symbol),

$$
\alpha=\sqrt{(}\left(N_{t}-1\right)
$$

substituting back in Eqn.(17), theresult follows.

\section{APPENDIX B}

PROOF OF (29)

Deriving Eq.(28) from Eq.(22)

$$
\wedge\left(b_{n}\right)=\log \left[\frac{\sum p\left(z \mid b_{n}=\beta_{1}\right)}{\sum p\left(z \mid b_{n}=\beta_{0}\right)}\right]=\log \left[\frac{\sum \frac{1}{\sqrt{2 \pi \sigma_{\nu}}} \frac{-\left(z-\alpha \beta_{1}\right)^{2}}{\nu^{2}}}{\sum \frac{1}{\sqrt{2 \pi \sigma_{\nu}}} \frac{-\left(z-\alpha \beta_{0}\right)^{2}}{\nu^{2}}}\right]
$$

For a 16QAM constellation, we have 4-amplituude $(\beta)$ levels which are $-3 \beta,-\beta, \beta$ and $3 \beta$. By substituting the amplitude values in the expression we have

$$
\wedge\left(b_{0}\right)=\log \left[\frac{\exp \frac{-(z-3 \alpha \beta)^{2}}{\nu^{2}}+\exp \frac{-(z-\alpha \beta)^{2}}{\nu^{2}}}{\exp \frac{-(z+3 \alpha \beta)^{2}}{\nu^{2}}+\exp \frac{-(z+\alpha \beta)^{2}}{\nu^{2}}}\right] .
$$

Performing a piece wise computation, we have

$$
\begin{aligned}
& \wedge\left(b_{0}\right)=\log \left[\frac{\exp \frac{-(z-\alpha \beta)^{2}}{\nu^{2}}}{\exp \frac{-(z+3 \alpha \beta)^{2}}{\nu^{2}}}\right] \quad z<-2 \beta \\
& =\log \left[\frac{\exp \frac{-(z-\alpha \beta)^{2}}{\nu^{2}}}{\exp \frac{-(z+\alpha \beta)^{2}}{\nu^{2}}}\right] \quad-2 \beta \leq z<2 \beta \\
& =\log \left[\frac{\exp \frac{-(z-3 \alpha \beta)^{2}}{\nu^{2}}}{\exp \frac{-(z+\alpha \beta)^{2}}{\nu^{2}}}\right] \quad-2 \alpha \leq z>2 \beta \\
& \wedge\left(b_{0}\right)=4 \beta \begin{cases}\frac{\alpha}{\nu^{2}}(2 z+2 \alpha \beta) & z<-2 \beta \\
\frac{\alpha}{\nu^{2}}(z) & -2 \alpha \beta z<2 \beta \\
\frac{\alpha}{\nu^{2}}(2 z-2 \alpha \beta) & z>2 \beta\end{cases} \\
& \wedge\left(b_{1}\right)=\log \left[\frac{\exp \frac{-(z+\alpha \beta)^{2}}{\nu^{2}}}{\exp \frac{-(z+3 \alpha \beta)^{2}}{\nu^{2}}}\right] \quad z<0 \\
& \wedge\left(b_{1}\right)=\log \left[\frac{\exp \frac{-(z-\alpha \beta)^{2}}{\nu^{2}}}{\exp \frac{-(z-3 \alpha \beta)^{2}}{\nu^{2}}}\right] \quad z>0 \\
& \wedge\left(b_{1}\right)=4 \beta \begin{cases}\frac{\alpha}{\nu^{2}}(z+2 \alpha \beta) & z<0 \\
\frac{\alpha}{\nu^{2}}(-z+2 \alpha \beta) & z>0 .\end{cases}
\end{aligned}
$$

By approximating the piecewise functions above, $\wedge\left(b_{0}\right)$ and $\wedge\left(b_{1}\right)$ can be expressed as

$$
\begin{gathered}
\wedge\left(b_{0}\right)=\frac{\alpha}{\nu^{2}}(z) \\
\wedge\left(b_{1}\right)=\frac{\alpha}{\nu^{2}}(-|z|+2) .
\end{gathered}
$$

\section{REFERENCES}

[1] L. Wen, R. Razavi, M. A. Imran, and P. Xiao, "Design of joint sparse graph for ofdm system," IEEE Transactions on Wireless Communications, vol. 14, no. 4, pp. 1823-1836, April 2015.

[2] P. Xiao, C. Toal, D. Burns, V. Fusco, and C. Cowan, "Transmit and receive filter design for ofdm based wlan systems," in Wireless Communications and Signal Processing (WCSP), 2010 International Conference on, Oct 2010, pp. 1-4.

[3] L. Zhang, P. Xiao, and A. Quddus, "Cyclic prefix-based universal filtered multicarrier system and performance analysis," IEEE Signal Processing Letters, vol. 23, no. 9, pp. 1197-1201, Sept 2016.

[4] L. Zhang, A. Ijaz, P. Xiao, A. Quddus, and R. Tafazolli, "Single-rate and multi-rate multi-service systems for next generation and beyond communications," IEEE PIMRC, 2016, 2016.

[5] R. Razavi, P. Xiao, and R. Tafazolli, "Information theoretic analysis of ofdm/oqam with utilized intrinsic interference," IEEE Signal Processing Letters, 2015.

[6] J. Du, P. Xiao, J. Wu, and Q. Chen, "Design of isotropic orthogonal transform algorithm-based multicarrier systems with blind channel estimation," IET Communications, vol. 6, no. 16, pp. 2695-2704, 2012.

[7] S. H. Muller-Weinfurtner, "Coding approaches for multiple antenna transmission in fast fading and ofdm," IEEE Transactions on Signal Processing, vol. 50, 2002.

[8] "IEEE standard for information technology- telecommunications and information exchange between systemslocal and metropolitan area networks- specific requirements-part 11: Wireless lan medium access control (mac) and physical layer (phy) specifications-amendment 4: Enhancements for very high throughput for operation in bands below 6 ghz." IEEE P802.11ac/D5.0, January 2013, pp. 1-440, 2013.

[9] J. Wang, O. Y. Wen, and S. Li, "Soft-output mmse mimo detector under imperfect channel estimation," in 2008 IEEE Wireless Communications and Networking Conference, 2008, pp. 1334-1338.

[10] I. B. Collings, M. R. G. Butler, and M. McKay, "Low complexity receiver design for mimo bit-interleaved coded modulation," in Spread Spectrum Techniques and Applications, 2004 IEEE Eighth International Symposium on, 2004, pp. 12-16.

[11] N. K. Chavali and A. R. Yalla, "A soft-demapper for coded mimoofdm system," in Contemporary Computing and Informatics (IC3I), 2014 International Conference on, 2014, pp. 451-457.

[12] L. M. A. Jalloul, S. P. Alex, and M. M. Mansour, "Soft-output mimo detectors with channel estimation error," IEEE Signal Processing Letters, vol. 22, no. 7, pp. 993-997, 2015.

[13] P. Xiao and R. Liu, "Multi-user detector for multi-carrier cdma systems," IET Electronic Letters, 2008.

[14] F. Tosato and P. Bisaglia, "Simplified soft-output demapper for binary interleaved cofdm with application to hiperlan/2," in Communications, 2002. ICC 2002. IEEE International Conference on, vol. 2, 2002, pp. $664-668$ vol.2.

[15] P. Xiao, C. Cowan, V. Fusco, T. Ratnarajah, and A. Fagan, "Soft demapping for ieee 802.11 ofdm system," 2010.

[16] J. Mao, M. A. Abdullahi, P. Xiao, and A. Cao, "A low complexity 256qam soft demapper for 5g mobile system," Proc. EuCNC 2016, Athens, Greece, 2016.

[17] X. Sun and Z. Zeng, Advances in Computer Science and Education Applications: International Conference, CSE 2011, Qingdao, China, July 9-10, 2011. Proceedings, Part II. Berlin, Heidelberg: Springer Berlin Heidelberg, 2011, ch. A Novel Simplified Log-Likelihood Ratio for Soft-Output Demapping of CMMB, pp. 350-356. [Online]. Available: http://dx.doi.org/10.1007/978-3-642-22456-0_51

[18] B. O'Hara and A. Petrick, The IEEE 802.11 Handbook: A Designer's Companion. Standards Information Network IEEE Press, 1999. 